\title{
Refinación de la Fase Glicerina del Biodiesel de Aceite de Palma empleando Ácidos Minerales
}

\author{
Fernando Cardeño, Luis J. Gallego y Luis A. Rios \\ Grupo Procesos Fisicoquímicos Aplicados, Universidad de Antioquia, Sede de Investigación \\ Universitaria, Cra. 53 \# 61 - 30, Medellín-Colombia. (e-mail: efecl797@yahoo.com)
}

Recibido Mar. 22, 2011; Aceptado May. 05, 2011; Versión Final recibida May. 12, 2011

\begin{abstract}
Resumen
Se estudió un método de purificación para la glicerina procedente de la producción de biodiesel de aceite de palma, para alcanzar los estándares requeridos para su uso en aplicaciones que no requieran el grado USP (United States Pharmacopoeia), definidos por las normas técnicas colombianas. Se usó diseño factorial $2^{3}$ con tres variables: tipo de ácido (fosfórico o sulfúrico), cantidad de metanol y $\mathrm{pH}$. Se encontró que el tratamiento con ácido sulfúrico, con $50 \%$ de metanol y $\mathrm{pH}$ de 6 , es el que da mejores resultados y que se requiere una decoloración con carbón activado para cumplir con la exigencia de color. La glicerina refinada y decolorada obtenida cumple con los requerimientos de la glicerina tipo 2 grado 1 y 2 según la Norma Técnica colombiana NTC1274.
\end{abstract}

Palabras clave: biodiesel, glicerina, purificación, ácidos minerales, normas colombianas

\section{Refining of Glycerin Phase from Palm Oil Biodiesel using Mineral Acids}

\begin{abstract}
A method is proposed for the purification of glycerin from palm oil biodiesel production to achieve the required standards, for use in applications non-requiring USP grade (United States Pharmacopoeia), particularly those defined by Colombian standards. A factorial design $2^{3}$ was used with the three variables: type of acid (phosphoric or sulfuric acid), quantity of methanol and $\mathrm{pH}$. It was found that the treatment with sulfuric acid, $50 \%$ methanol and $\mathrm{pH}$ of 6 gave the best results, and requires an activated carbon bleaching to meet the required color. Refined and bleached glycerin obtained meets the requirements of glycerin type 2 grades 1 and 2 according to the Colombian standard NTC1274.
\end{abstract}

Keywords: biodiesel, glycerin, palm oil, mineral acids, Colombian standard 


\section{INTRODUCCIÓN}

La industria oleoquímica mundial ha venido teniendo una importante expansión en los últimos años, principalmente en la producción de los biocombustibles. La producción de biodiesel, por ejemplo, se ha venido incrementado debido a las perspectivas de agotamiento de los combustibles fósiles, así como de una preocupación medioambiental por las emisiones de gases contaminantes como $\mathrm{CO}, \mathrm{CO}_{2}, \mathrm{SOx}$ y $\mathrm{NOx}$ asociados a la quema de hidrocarburos como gasolina y diesel. El biodiesel presenta ventajas en estos temas, pues es un combustible que ha demostrado ser relativamente más limpio y que en su producción se utilizan recursos renovables (Sharma YC et al, 2008; Luque et al, 2008a) En Colombia dicho incremento de la producción se ha visto favorecido por algunos beneficios tributarios como la exención de algunos impuestos nacionales e internacionales y los estímulos para la producción de aceite de palma por instituciones como FEDEPALMA.

La producción de biodiesel a partir de aceites vegetales genera glicerol como subproducto como se muestra en la Fig. 1; esta reacción es una simplificación de tres reacciones reversibles y consecutivas en las cuales se forman mono y diglicéridos como productos intermedios. Se estima que por cada kilogramo producido de biodiesel se generan aproximadamente 0,1 kilogramos de glicerol. Es fundamental contar con un aceite que tenga una acidez muy baja para garantizar unos productos de la reacción de transesterificación que no presenten problemas en la etapa de separación de fases por formación de jabones (reacciones de saponificación) que actúan como tensoactivos (Echeverri et al, 2011; Cardeño et al., 2010).

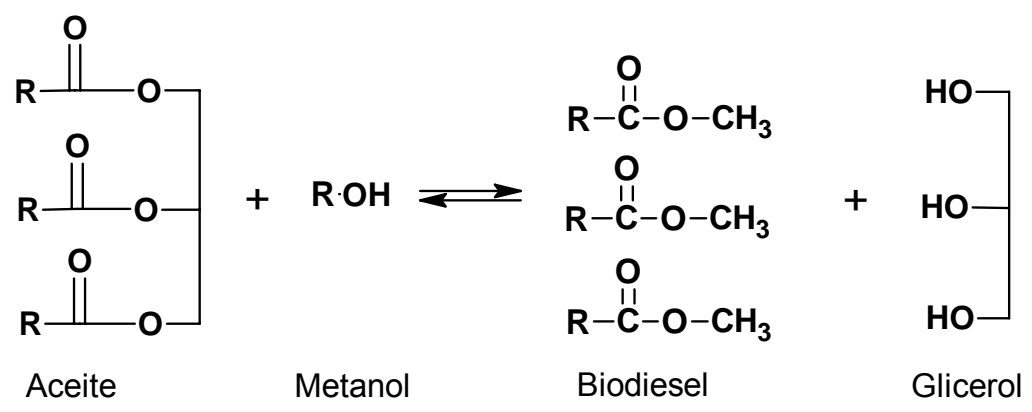

Fig. 1: Reacción de transesterificación.

Debido a que se proyecta un aumento en la demanda de biodiesel, el aprovechamiento de la glicerina se convierte pues en un tema de suma importancia, puesto que con ello se mejora notablemente la economía global del proceso de producción de biodiesel. La glicerina tiene diversas aplicaciones industriales, por ejemplo en la fabricación de resinas alquídicas, como plastificante en adhesivos y para la fabricación de explosivos (Shahidi, 2005); también algunos autores emplean la glicerina cruda proveniente de la producción de biodiesel para la síntesis de mono y diglicéridos (Echeverri et al, 2011). Recientemente se han propuesto nuevas aplicaciones como su uso en aditivos oxigenados para combustibles, como triacetina (Luque et al, 2008b) y cetales (Giraldo et al, 2009); la transformación en otros compuestos de mayor valor como proopanodiol y acroleína (Mota et al, 2009), esteres de glicerol, glicerol carbonato, y sus uso como reactivo para gasificación y producción de gas de síntesis (Behr et al, 2008). Así pues, el principal desafío que se tiene es la purificación y adecuación de la glicerina para aplicaciones actuales y futuras, de tal forma que su uso sea económico, técnico y ambientalmente viable. Tradicionalmente la glicerina se purifica mediante destilación con alto vacio, el cual es un proceso de alta demanda energética y que produce glicerina de alta pureza (USP); sin embargo, para algunas aplicaciones en la industria no es necesaria esta glicerina de alta pureza (Thompson y He, 2006; Diaz y Miller, 1987; Thomas et al., 1991; Bam et al., 1995; Hazimah et al., 2000; OOl et al., 2001; Yong et al., 2001a; Yong et al., 2001b; Aiken, 2006).

Esta investigación buscó fundamentalmente estudiar y proponer un método de purificación para la glicerina procedente de la producción de biodiesel, de tal forma que se alcancen los estándares requeridos para su uso, en aplicaciones que no requieran el grado USP, particularmente los 
definidos por las Normas Técnicas Colombianas (Miner y Weil, 1949; NTC 1274, 1980; NTC 700, 1980; NTC 689, 1980; NTC 1039, 1980); estas normas NTC están fundamentadas en otras normas internacionales tales como la ISO 2879, la Norma Británica BS 2621 - 5 y la norma americana (ASTM D-1257, 2007).

\section{MATERIALES Y MÉTODOS}

Hay seis aspectos de importancia relacionados con los materiales y los métodos empleados en este estudio: los reactivos usados, la etapa de transesterificación; el acondicionamiento de la fase glicerina; el proceso de purificación de la fase glicerina; el diseño experimental y el proceso de decoloración. Estos se describen en lo que sigue.

\section{Reactivos}

El metanol usado fue grado industrial. El ácido sulfúrico empleado fue grado analítico (98\% MERCK), el ácido fosfórico fue grado analítico (85\% JT Baker). El $\mathrm{NaOH}$ fue grado analítico (MERCK). El carbón activado para la decoloración fue grado analítico (Darco G60 granular malla 325-JT Baker).

\section{Etapa de transesterificación}

Se usaron las condiciones siguientes: Cantidad de catalizador $(\mathrm{NaOH} 0.7 \%)$, temperatura $\left(60^{\circ} \mathrm{C}\right.$, tiempo (1 hora).agitación (300 rpm) y relación molar aceite/alcohol (1/6 y 1/9). La reacción se llevó a cabo disolviendo el catalizador en el metanol mediante agitación, para luego incorporarlo al aceite. Se sometió esta mezcla condiciones de temperatura y agitación indicadas. Terminado el tiempo de reacción, los productos se almacenan en un embudo de decantación para obtener una separación de las dos fases; la fase más pesada es la Fase Glicerina y la más liviana es la fase biodiesel; Fase glicerina (FG) en este trabajo hace referencia al subproducto más denso obtenido en la producción de biodiesel y constituido por glicerol, metanol, catalizador y trazas de metilésteres, glicéridos y agua principalmente. Al final de la reacción de transesterificación el metanol en exceso se distribuye en las dos fases.

\section{Acondicionamiento de la Fase Glicerina}

La FG fue obtenida de acuerdo a procesos estándares empleados en plantas de producción de biodiesel y también ampliamente utilizados en la literatura a nivel de laboratorio (Echeverri et al, 2011). La FG fue previamente sometida a un proceso de evaporación a $85^{\circ} \mathrm{C}$ para recuperar el metanol residual de la transesterificación; el producto de este tratamiento fue una fase de color café, de consistencia pastosa, y con algún contenido de líquido; esta nueva fase la denominamos FGSM. Esta evaporación comúnmente se hace en las plantas productoras de biodiesel para recuperar el alcohol y reciclarlo en la etapa de transesterificación.

\section{Proceso de purificación de la fase glicerina}

El proceso de purificación se resume en la Fig. 2. Obsérvese que en la primera etapa de este proceso nuevamente se adiciona metanol al sistema (FGSM); sin embargo en la práctica sólo será necesario ajustar la cantidad de metanol adecuada para el proceso de purificación.

Después de diluir la FGSM con metanol se realizó un proceso de neutralización tomando como punto de referencia un diseño experimental $2^{\mathrm{K}}$ en el que se evaluaron 3 variables: $\mathrm{pH}$, cantidad de metanol y tipo de ácido (fosfórico y sulfúrico). Posteriormente se realizaron operaciones de filtración y evaporación a la FGSM ya transformada; el nuevo producto obtenido lo denominamos Glicerina Refinada (GR) y el cual fue caracterizado de acuerdo a la Norma Técnica Colombiana NTC 1274; finalmente, se realizó una etapa de decoloración al producto obtenido el cual definimos como Glicerina Refinada Decolorada (GRD). Para la etapa de neutralización se toma una cantidad conocida de la FGSM, se mide la cantidad de metanol a añadir y se le incorpora con muy buena agitación a temperatura ambiente. 


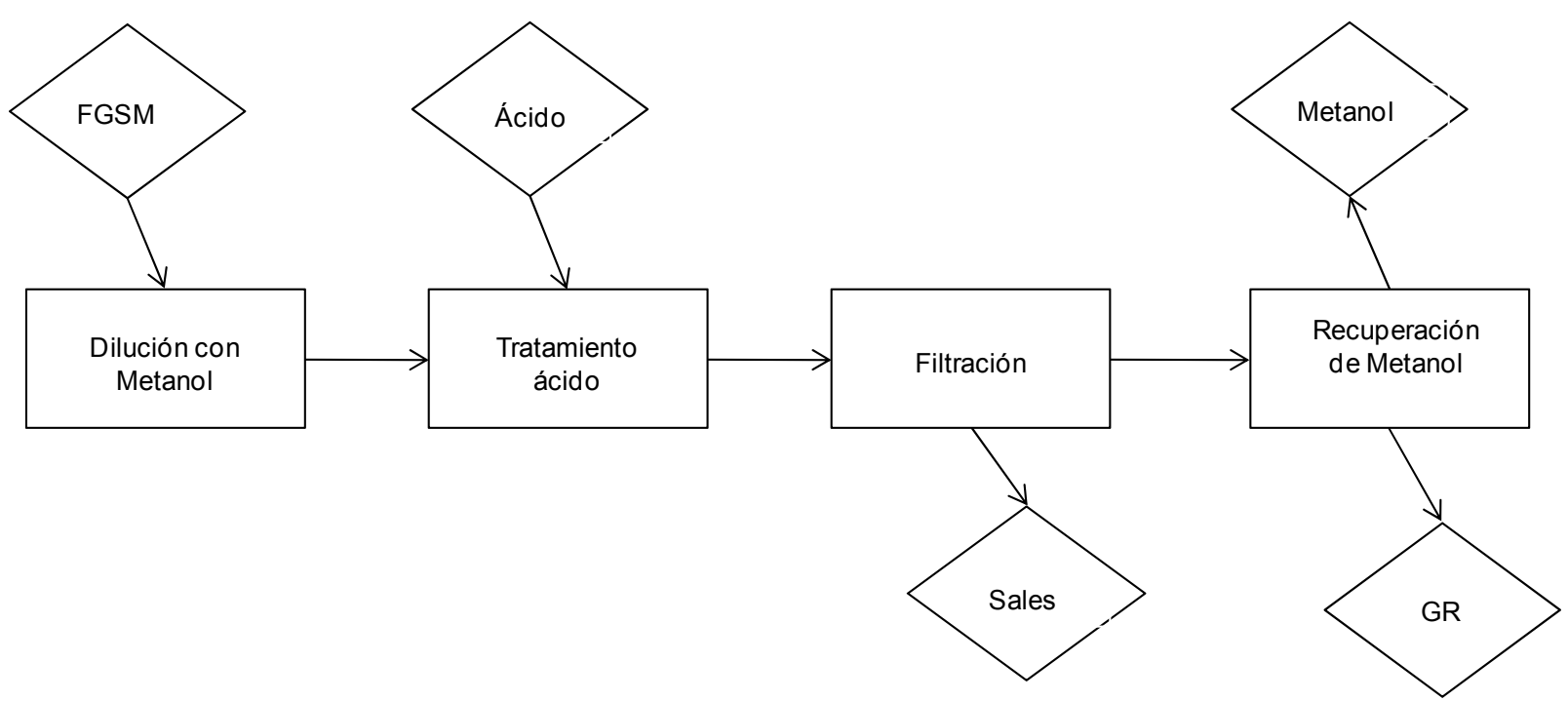

Fig. 2: Proceso de purificación de la fase glicerina.

Luego se añade el ácido de manera controlada, mientras se observa que el pH de la solución sea el adecuado, la tolerancia que se usó para determinar si el pH es el requerido es de más o menos 0,2 unidades; es necesario este control ya que la solución tiene baja capacidad amortiguadora a los $\mathrm{pH}$ que se están trabajando. De acuerdo al diseño experimental propuesto, se realizaron un total de 8 experimentos por duplicado como se muestra en la tabla 1 y tabla 2. Después del tratamiento ácido y usando las condiciones mostradas en la tabla 1 y tabla 2, se procedió a filtrar la solución a través de papel de filtro Advantec \#1. En el filtro quedan retenidas las sales formadas, así como una parte de la materia orgánica que no es glicerol. El filtrado resultó ser un líquido viscoso de color café claro rojizo, constituido principalmente por glicerol y metanol mientras que la torta de filtración es un sólido pastoso color claro, constituida principalmente por sales inorgánicas y residuos orgánicos (jabones, ácidos grasos, glicéridos etc.). Luego de la filtración se procedió a separar el metanol de la glicerina, lo cual se hizo mediante rotoevaporación a $95^{\circ} \mathrm{C}$ y vacío. Finalmente se obtuvo un metanol recuperado y la GR.

\section{Diseño experimental}

Se llevó a cabo un diseño factorial $2^{3}$; como se muestra en la tabla 1; los niveles se escogieron de acuerdo a los siguiente lineamientos: para el $\mathrm{pH}$ se escogieron estos valores debido a que en la hidrólisis de los jabones de ácidos grasos se requiere un $\mathrm{pH}$ neutro o ácido, pero como la acidez es una característica regulada en la glicerina el $\mathrm{pH}$ no puede disminuirse demasiado; el contenido de metanol se fijó de acuerdo al contenido de metanol que tiene la fase glicerina cuando se emplean diferentes relaciones molares aceite/metanol; experimentalmente en la reacción de transesterificación, se evidenció que para una relación aceite/metanol de 1/6 y 1/9 se obtienen Fases Glicerina con aproximadamente $40-50 \%$ y $60-70 \%$ de metanol respectivamente, lo cual depende principalmente de los productos obtenidos después de la reacción de transesterificación y también del tiempo de decantación de las fases biodiesel y glicerina.

El análisis de contenido de glicerol, contenido de éster metílico, contenido de mono y diglicéridos se realizó en un equipo AGILENT 7890A. El color se midió utilizando un espectrofotómetro, de acuerdo al procedimiento para medir el color del glicerol reportado por Miner y Weil, 1949. En la determinación de cenizas, se usó una mufla Memmert y se adoptó el procedimiento reportado en la norma NTC 700. La determinación de humedad fué encontrada empleando el método KarlFisher usando un titulador automático Metrohm. Las pruebas de contenido de acidez y de equivalente de ácido grasos se realizaron conforme a la norma NTC 1039, y usando para las valoraciones un titulador automático Metrohm. 
Tabla 1. Variables de entrada empleadas en el proceso de neutralización ácida.

En la Tabla, -1 corresponde a sulfúrico y 1 a fosfórico.

\begin{tabular}{|l|c|c|}
\hline \multicolumn{1}{|c|}{ Factores } & Nivel mínimo & Nivel máximo \\
\hline Cantidad de metanol (\% peso) & $50 \%$ & $70 \%$ \\
\hline $\mathrm{pH}$ & 6 & 7 \\
\hline Tipo ácido* & 1 & -1 \\
\hline
\end{tabular}

Tabla 2. Diseño factorial para el proceso de neutralización ácida.

\begin{tabular}{|c|c|c|c|}
\hline$\#$ & Tipo de ácido & Cantidad de metanol (\% másico) & $\mathrm{pH}$ \\
\hline 1 & Fosfórico & 50 & 6 \\
\hline 2 & Fosfórico & 50 & 7 \\
\hline 3 & Fosfórico & 70 & 6 \\
\hline 4 & Fosfórico & 70 & 7 \\
\hline 5 & Sulfúrico & 50 & 6 \\
\hline 6 & Sulfúrico & 50 & 7 \\
\hline 7 & Sulfúrico & 70 & 6 \\
\hline 8 & Sulfúrico & 70 & 7 \\
\hline
\end{tabular}

\section{Proceso de decoloración}

Adicionalmente a los productos con los mejores resultados derivados del tratamiento ácido se les realizó un tratamiento con carbón activado comercial para mejorar su color. Las condiciones a utilizar en esta etapa no se modificaron y se utilizaron condiciones ya reportadas en la literatura (Bailey's, 2005) para procesos de decoloración similares (véase tabla 3).

Tabla 3: Condiciones para la decoloración.

\begin{tabular}{|l|c|}
\hline \multicolumn{1}{|c|}{ Parámetro } & Valor \\
\hline Cantidad utilizada & $5 \%$ másico \\
\hline Tiempo de contacto & 1 hora \\
\hline Temperatura & $90^{\circ} \mathrm{C}$ \\
\hline
\end{tabular}

\section{RESULTADOS Y DISCUSIÓN}

\section{Caracterización de la Fase Glicerina}

A la FGSM se le realizó medición de contenido de glicerol, contenido de éster metílico, jabones, humedad, catalizador y también contenido de mono y diglicéridos (véase tabla 4).

Tabla 4. Caracterización FGSM.

\begin{tabular}{|l|l|c|}
\hline \multicolumn{1}{|c|}{ Sustancia } & \multicolumn{1}{|c|}{ Técnica empleada } & FGSM \\
\hline Metilésteres (\% p/p) & Cromatografía gaseosa (BS EN 14103:2003) & 0.03 \\
\hline Glicerol (\% p/p) & $\begin{array}{l}\text { Cromatografía gaseosa (ASTM D 6584-07, 2007; Brüschweiler y } \\
\text { Dieffenbacher, 1991) }\end{array}$ & 84.60 \\
\hline Monoglicéridos (\% p/p) & $\begin{array}{l}\text { Cromatografía gaseosa (ASTM D 6584-07, 2007; Brüschweiler et al., } \\
1991)\end{array}$ & 0.22 \\
\hline Diglicéridos (\% p/p) & $\begin{array}{l}\text { Cromatografía gaseosa (ASTM D 6584-07, 2007; Brüschweiler et al., } \\
1991) .\end{array}$ & 0.09 \\
\hline Jabones (\% p/p) & AOCS method Cc 17-79 (Van Gerpen et al., 2004).. & 4.30 \\
\hline Catalizador (\% p/p) & Titulación HCl. & 5.17 \\
\hline Agua (\% p/p) & Karl Fisher Titration (ASTM E 203-01) & 4.82 \\
\hline Otros (\% p/p) & Metanol, triglicéridos, sales, trazas de base o ácido & 0.77 \\
\hline $\begin{array}{l}\text { Arsénico, expresado } \\
\text { como As, en ppm máx. }\end{array}$ & $\begin{array}{l}\text { El método se basa en la reacción entre el dietil-ditiocarbamato de } \\
\text { plata y la arsina (NTC 1274) }\end{array}$ & 0.04 \\
\hline
\end{tabular}




\section{Caracterización de la Glicerina Refinada}

Los resultados de la caracterización para la GR se muestran en la tabla 5 . Se define como variable fundamental de salida el contenido de cenizas pues este es un indicador de la efectividad del tratamiento (indica de manera indirecta el contenido remanente de catalizador); sin embargo, a todos los productos también se les midió contenido de glicerol como se muestra.

Tabla 5. Resultados contenido de glicerol y cenizas.

\begin{tabular}{|c|c|c|c|}
\hline Exp. \# & Cenizas (\% peso) & Glicerol (\% peso) & Humedad (\%peso) \\
\hline 1 & $4,490+/-0.007$ & $94,2+/-0.1$ & $2,6+/-0.11$ \\
\hline 2 & $2,760+/-0.009$ & $84,5+/-0.1$ & $11+/-0.12$ \\
\hline 3 & $1,980+/-0.006$ & $95,5+/-0.1$ & $1,1+/-0.10$ \\
\hline 4 & $1,830+/-0.008$ & $88,9+/-0.1$ & $7,8+/-0.10$ \\
\hline 5 & $0,331+/-0.002$ & $96,0+/-0.1$ & $2,7+/-0.10$ \\
\hline 6 & $0,572+/-0.002$ & $95,7+/-0.1$ & $2,7+/-0.11$ \\
\hline 7 & $0,643+/-0.004$ & $91,9+/-0.1$ & $3,8+/-0.10$ \\
\hline 8 & $0,816+/-0.003$ & $96,5+/-0.1$ & $2,0+/-0.11$ \\
\hline
\end{tabular}

Se observa que el experimento 5 presenta los mejores resultados en cuanto al contenido de cenizas; finalmente este producto (experimento 5), se sometió a un proceso adicional de decoloración: primero se calentó a 90 grados centígrados y se le adicionó $5 \%$ en peso de carbón activado, se dejó el sistema en contacto con agitación durante 1 hora a la temperatura establecida. Finalmente se filtró para separar el carbón activado y se obtuvo la GRD, que se sometió a evaporación a condiciones más drásticas (130 grados y 0,5 atmósferas de vacío) para eliminar agua; es de anotar, que este proceso de decoloración solo se realizó con los productos obtenidos con $\mathrm{H}_{2} \mathrm{SO}_{4}$ pues fue el que mejores resultados presentó. En la tabla 6 se reporta la caracterización de la mejor GR y GRD obtenida (experimento 5) y que se pueden comparar con las características exigidas por las normas técnicas colombianas para la glicerina tipo 2 (grado 1) y tipo 1 (grado 4) que son las más próximas obtenidas a la glicerina obtenida (NTC 1274, 1980, referenciada por Norma británica BS 2621 - 5 Specifications for Glycerol).

La NTC 1274 establece que las cenizas totales obtenidas para los productos sólo alcanzan a cubrir los requerimientos del glicerol tipo 2; para reducir el contenido de cenizas a un valor menor al máximo permitido se puede llevar a cabo un procedimiento alterno que favorezca la precipitación de las sales, como el uso de otros solventes o de otras técnicas como es el empleo de resinas de intercambio iónico.

Se observa que el mejor ácido para el proceso de neutralización es el ácido sulfúrico (experimentos 5 a 8), puesto que todos los experimentos llevados a cabo con este ácido fueron más satisfactorios que con el ácido fosfórico (experimentos 1 a 4). Esto se debe a que las sales de fosfato presentan una mayor solubilidad en el sistema metanol/glicerina, quedando solubilizadas en el sistema y siendo imposibles de remover por filtración. Para este tipo de procesos, en los que se involucra la adición de un solvente, deben tenerse en cuenta otros factores como el consumo de materia prima y el incremento del volumen a tratar, con los que se incrementan los costos de bombeo y transporte, así como la necesidad de usar equipos de mayor tamaño.

La solubilidad de las sales viene afectada tanto por el tipo de solvente como por la cantidad de este. Para nuestro caso en particular vemos que la cantidad óptima difiere según el tipo de sal formada, para el sulfato con el $50 \%$ ya se alcanzó la mínima solubilidad observada de esta en el sistema, mientras que para el fosfato a $70 \%$ se obtuvo un resultado mejor posiblemente debido a que su diferencia de solubilidad entre el metanol y la glicerina es más marcada, posiblemente a mayor cantidad de metanol se podrían alcanzar menores solubilidades del fosfato. 
Tabla 6. Resultados comparativos. En la Tabla, A: son unidades amarillas, $\mathrm{R}$ son unidades rojas ** Equivalente de $\mathrm{NaOH} \% \mathrm{p} / \mathrm{p}$

\begin{tabular}{|l|c|c|c|c|}
\hline \multicolumn{1}{|c|}{ Índice } & $\begin{array}{c}\text { Valor GR } \\
\text { Exp. 5 }\end{array}$ & $\begin{array}{c}\text { Valor GRD } \\
\text { Exp. 5 }\end{array}$ & $\begin{array}{c}\text { Valor exigido norma } \\
\text { Glicerina tipo 2 grado 1 } \\
\text { (NTC 1274, 1980). }\end{array}$ & $\begin{array}{c}\text { Valor exigido norma } \\
\text { Glicerina tipo 1 grado 4 } \\
\text { (NTC 1274, 1980). }\end{array}$ \\
\hline Color & 180A. 29R & $\begin{array}{c}0.44 \mathrm{~A} \\
0.00 \mathrm{R}\end{array}$ & $\mathrm{N} / \mathrm{A}$ & $\begin{array}{c}2.0 \mathrm{R}, 7.5 \mathrm{~A} \text { (Escala } \\
\text { Lovibond) }\end{array}$ \\
\hline Cenizas (\% masa) & 0.331 & 0.28 & 1.0 & $\begin{array}{c}0.01 \text { (como cenizas } \\
\text { sulfatadas) }\end{array}$ \\
\hline Acidez $\left(\% \mathrm{Na}_{2} \mathrm{O}\right)$ & 0.038 & 0.011 & $\mathrm{~N} / \mathrm{A}$ & 0.01 \\
\hline Humedad $(\%$ masa) & 2.65 & 2.8 & $\mathrm{~N} / \mathrm{A}$ & $\mathrm{N} / \mathrm{A}$ \\
\hline $\begin{array}{l}\text { Contenido de ácidos } \\
\text { grasos } * \text { (\% masa) }\end{array}$ & 0.15 & 0.010 & $\mathrm{~N} / \mathrm{A}$ & 98 \\
\hline $\begin{array}{l}\text { Contenido de Glicerol } \\
\text { (\% masa) }\end{array}$ & 96.0 & 96.60 & 88 & $\mathrm{~N} / \mathrm{A}$ \\
\hline $\begin{array}{l}\text { Arsénico, expresado } \\
\text { como As, en ppm máx. }\end{array}$ & negativo & negativo & 2.0 & \\
\hline
\end{tabular}

Con relación a la dependencia del pH en el tratamiento ácido, esta variable afecta la solubilidad de las sales: para el sulfato de sodio, un pH ácido favorece la precipitación ya que el ión sulfato y el bisulfato son bases muy débiles, con mínimos de solubilidad a $\mathrm{pH}$ ácidos. El fosfato al tener un carácter básico un poco más marcado no favorece su solubilidad a $\mathrm{pH}$ ácidos, y sus mínimos de solubilidad se encuentran a pH neutros o alcalinos, explicándose así la razón por la cual los experimentos con $\mathrm{pH}$ de 6 arrojan mejores resultados para el ácido sulfúrico, mientras que para el ácido fosfórico se observa un comportamiento contrario. Cabe aclarar que el pH no puede llevarse a extremos pues el producto final (glicerina) tiene unas limitaciones en cuanto a la acidez o basicidad presente en él.

El contenido de humedad en los productos señala que se requiere un proceso de purificación más riguroso que permita retirar mayor contenido de agua de las muestras como puede ser el uso de agentes deshidratantes; otro factor importante podría abarcar la manipulación y almacenamiento de las muestras, debido a que la glicerina es higroscópica. Del mismo modo, comparando los resultados obtenidos para el contenido de glicerol, se puede justificar en gran parte la dependencia de estos resultados con la humedad, así que la consideración de esta variable se hace importante a la hora de aplicar a los requerimientos establecidos por la norma NTC 1274.

La GR y la GRD obtenidas en esta investigación pueden encontrar aplicaciones directas como puede ser la síntesis de mono y di glicéridos para la producción de resinas alquídicas; también puede ser usado como humectante industrial. En aplicaciones para alimentación animal se deberá hacer un estudio más detallado en cuanto a los contenidos mínimos de metanol y arsénico que eventualmente puede quedar luego de cualquier proceso de purificación de la fase glicerina procedente del proceso de producción de biodiesel.

\section{CONCLUSIONES}

El proceso implementado para la refinación de la fase glicerina, procedente del proceso de producción de biodiesel, es efectivo. Se encontró que el tratamiento con $\mathrm{H}_{2} \mathrm{SO}_{4}, 50 \%$ de metanol y $\mathrm{pH}$ de 6 es el mejor, y que se requiere una decoloración con carbón activado para cumplir con la exigencia de color.

El contenido de humedad en los productos señala que se requiere un proceso de purificación más riguroso que permita retirar mayor contenido de agua de las muestras. Se puede considerar exitoso el proceso de purificación. En términos generales se logró remover de manera importante 
los contaminantes presentes en la fase glicerina con métodos relativamente simples. Por ejemplo el parámetro cenizas totales se logró disminuir en cerca del $95 \%$.

Si bien el producto obtenido no alcanza los grados de glicerina refinada, se adecua con holgura a los requisitos de glicerina cruda. Según la norma NTC 1274 la GRD obtenida cumple con los requerimientos de la glicerina tipo 2 grados 1-2.

El alcance de los grados más puros de glicerina no puede lograrse con este tipo de tratamientos únicamente, y si se desea lograr los estándares de USP por ejemplo, se deben combinar procesos como el descrito en este trabajo con otros más complejos como la destilación a presión reducida, el intercambio iónico, etc.

Debe hacerse énfasis en que el futuro del aprovechamiento industrial de la glicerina no es la obtención y utilización de un producto ultrapuro, sino el uso de la glicerina obtenida del biodiesel, con el menor tratamiento posible, que garantice el normal desempeño de un proceso en particular, y la obtención de un producto deseado dentro de los parámetros presupuestados para éste. En este orden de ideas, un tratamiento de purificación/adecuación debe abogar por su simpleza y por la obtención del mejor producto con la mínima utilización de energía y recursos. En consecuencia, consideramos que los métodos evaluados en este trabajo, se enmarcan dentro de dicho escenario, pues logran un producto que puede ser utilizado en varias aplicaciones, mediante un tratamiento que no usa demasiados recursos y que es bastante simple en lo referente a equipos, reactivos y etapas.

\section{AGRADECIMIENTOS}

Los autores agradecemos al Ministerio de Agricultura y Desarrollo Rural de Colombia (Proyecto de Transición de la Agricultura-PTA) y a la Universidad de Antioquia (programa Estrategia de Sostenibilidad de Grupos) por su apoyo financiero.

\section{REFERENCIAS}

Aiken Jhon. Purification of Glicerin. US 7.126.032 (2006).

ASTM E 203-08. Standard test method for water using volumetric Karl Fischer titration. American Society for Testing and Materials. West Conshohocken (2008).

ASTM D 6584-07. Standard test method for determination of free and total glycerin in B-100 biodiesel methyl esters by gas chromatography. American Society for Testing and Materials. West Conshohocken (2007).

ASTM D1257 - 07. Standard Specification for High-Gravity Glycerin. American Society for Testing and Materials. West Conshohocken (2007).

Bam, Narendra y otros cinco autores. Method for purifying alcohol esters. US 5.424.467 (1995).

Behr, Arno y otros cuatro autores. Improved utilisation of renewable resources: New important derivatives of Glicerol. Green Chem, Vol. 10, No 1, p. 13-30 (2008).

BS EN 14103:2003. Fat and oil derivatives-Fatty acid methyl esters (FAME)-determination of ester and linolenic acid methyl ester contents. European Committee for Standardization (CEN). Brussels (2003).

Buenemann, T. y otros tres autores, Process for Purifiying Crude Glicerol. US 4.990.695 (1991).

Brüschweiler $H$, y Dieffenbacher, A. Determination of mono and DG by capillary gas chromatography-results of a collaborative study and the standardized method. Pure Appl Chem 63:1153-1162. (1991). 
Cardeño, Fernando y otros dos autores. Production of crude palm oil biodiesel by heterogeneous catalysis. Revista Facultad de Ingeniería de la Universidad de Antioquia. No. 5, p.81-86. ISSN 0120-6230. Jan/March (2010).

Diaz, Z. y Miller, James H. Glycerine purification process. US 4.683.347 (1987).

Echeverri, David y otros dos autores. Glycerolysis of Soybean Oil with Crude Glycerol Containing Residual Alkaline Catalysts from Biodiesel Production. Journal of the American Oil Chemists' Society. Volume 88, No 4, p. 551-557(2011).

Giraldo Sandra y otros tres autores. Síntesis de Aditivos para Biodiesel a partir de Modificaciones Químicas de la Glicerina. Información Tecnológica Vol. 20 Nº6, p. 75-84 (2009).

Hazimah, A. y otros dos autores. Recovery of Glycerol and other Valuables from Glycerol Pitch. MPOB Information Series No. 89. Malaysian Palm Oil Board, Bangi. Pag. 1-5 (2000).

Luque, Rafael y otros siete autores. Biofuels: a technological perspective. Energy Environ. No 1, p. 542-564 (2008a).

Luque Rafael y otros tres autores. Glycerol transformations on polysaccharide derived mesoporous materials. Applied Catalysis B: Environmental. No 82, p. 157-162 (2008b).

Miner C. S. y Weil C. M. . Application of spectrophotometric methods to the determination of glycerine color. Journal of the American oil chemist society (1949).

Mota, Claudio y otros dos autores. Gliceroquímica: novos produtos e processos a partir da glicerina de produção de biodiesel. Quim. Nova, Vol. 32, No. 3, 639-648 (2009).

NTC 1274. Norma Técnica Colombiana Productos químicos: Glicerol (1980).

NTC 700. Norma Técnica Colombiana. Glicerol: determinación de cenizas (1980).

NTC 689. Norma técnica Colombiana. Glicerol: determinación de la presencia de azúcar (1980).

NTC 1039. Norma técnica Colombiana. Glicerol: métodos de análisis (1980).

Ooi T. L y otros cuatro autores. Crude Glycerine Recovery from Glycerol Residue Waste from a Palm Kernel Oil Methyl Ester Plant. Journal of Oil Palm Research Vol.13(2). Pag. 16-22 (2001).

Shahidi, Fereidoon. Bailey's Industrial Oil and Fat Products. Volume 5, Edible Oil and Fat Products: Processing Technologies, 6th Edition John Wiley \& Sons (2005).

Sharma Y.C. y otros dos autores. Advancements in development and characterization of biodiesel: A review. Fuel; 87: 23552373 (2008).

Thompson J.J, y He B.B. Characterization of crude glycerol from biodiesel production from multiple feedstocks. Applied Engineering in Agriculture. Vol. 22(2): 261-265. (2006).

Van Gerpen, J y otros dos autores. Biodiesel Analytical Methods. National Renewable Energy Laboratory, Colorado. 2004.

Yong, K. C. y otros cuatro autores. Characterization of Glycerol Residue Generated from a Palm Kernel Oil Methyl Ester Plant. Journal of Oil Palm Research Vol. 13 (2). Pag. 1-6. (2001a).

Yong, K. C. y otros cuatro autores. Refining of Crude Glycerine Recovered from Glycerol Residue by Simple Vacuum Distillation. Journal of Oil Palm Research Vol. 13 (2). Pag. 39-44. (2001b). 
